Math. Model. Nat. Phenom.

Vol. 5, No. 2, 2010, pp. 1-4

\title{
Quantitative Neuroscience: From Chalk Board to Bedside
}

\author{
John G. Milton ${ }^{1}$ \\ W. H. Keck Science Center, The Claremont Colleges, Claremont, CA 91711, USA
}

Quantitative neuroscience, an interdisciplinary approach that brings together the mathematical, modeling, engineering and neuroscience communities, is rapidly bringing tangible hope of a better life to the tens of millions of people afflicted with diseases of the nervous system. The driving force is an increased understanding of how the nervous system exerts control and processes information.

In less than 15 years, quantitative neuroscience is seemingly doing the impossible $[12,17]$. Brain-computer interfaces now make it possible to translate thought into action $[6,7,8,15]$, replace lost limbs with robotic ones [9], prevent epileptic seizures [11, 16] and even to alleviate the symptoms of neuro-degenerative diseases, such as Parkinson's [14]. Is it possible to do even better?

The first theme of this issue explores two lines of mathematical research that have been particularly fruitful: 1) neural synchronization [19, 21], the fundamental process by which spatially distributed neural centers bind a sensory stimulus and coordinate their activities to respond to it, and 2) multistability [2, 10, 12], the concept that treatments may be possible by applying jolts of electricity to the right location in the brain at the right time $[11,16]$. Attention is drawn to the effects of time delays and random perturbations ("noise") on neural control and information processing. Since axonal conduction velocities are finite, time delays are an intrinsic component of all neural feedback loops. Stochastic effects arise from fluctuations in ion channels and quantal release and from the convergence of multiple independent synaptic inputs. Obviously the most promising mechanisms for neural processing are those which are robust in the presence of noise and delay. In other words, it is not only necessary to develop analytical expressions that describe neural synchronization regardless of the number of neurons [5], it is also necessary to understand the effects of noise of the dynamics of synchronizing neural populations [2]. Similarly the widespread occurrence of time-delayed feedback in neural pathways raises questions as to the role of time delays in information processing [10] and whether new effects arise from the interplay between noise

\footnotetext{
${ }^{1}$ E-mail: jmilton@jsd.claremont.edu
} 
and delay $[1,13]$.

The second theme explores the role of mathematics in verifying ideas at the bench top and the bedside. Attention is drawn to a number of interesting mathematical problems that traditionally have received little attention. For example, how should experimental protocols be designed to best optimize methods to estimate parameters [3]? An even more vexing question concerns the choice of the appropriate mesoscopic scale [14], i.e. the length scale at which one can approximate the statistical properties of the whole neural population without having to take into account the detailed properties of each of neuron. A major disconnect presently exists between computationally and experimentally oriented neuroscientists; whereas neuroscientists often focus on events at the molecular scale, modelers typically express their ideas in terms of membrane potential or neural spiking rates. Moreover those whose study the brain utilize indirect methods that either monitor neural metabolism (e.g. PET, fMRI) or the extracellular field potentials created by post-synaptic potentials on the large, vertically-oriented pyramidal neurons of neocortex (e.g. EEG, MEG). The next challenge for quantitative neuroscience will be to understand how these length scale boundaries can be crossed.

Clearly there is still much more to do before we can develop therapeutic devices where "both the cortex and the stimulator speak the same language" [4, 6, 14, 18, 20, 22]. However, the articles in this issue suggest that there is good reason for quantitative neuroscientists to share of the hope of patients that a better life is just around the corner.

\section{References}

[1] O. A. Åkerberg, M. J. Chacron. Noise shaping in neural populations with global delayed feedback. Math. Model. Nat. Phenom., 5 (2010), 100-124.

[2] R. E. L. de Ville, C. S. Peskin, J. H. Spencer. Dynamics of stochastic neuronal networks and the connections to random graph theory. Math. Model. Nat. Phenom., 5 (2010), $26-66$.

[3] D. Fairhurst, I. Tyukin, H. Nijmeijer, C. van Leeuwen. Observers for canonic models of coupled oscillators. Math. Model. Nat. Phenom., 5 (2010), 146-184.

[4] P. Fromhertz, A. Stent. Silicon-neuron junction: Capacitive stimulation of an individual neuron on a silicon chip. Phys. Rev. Lett., 75 (1995), 1670-1673.

[5] A. Garenne, J. Henry, C. O. Tarniceriu. Analysis of synchronization in a neural population by a population density approach. Math. Model. Nat. Phenom., 5 (2010), 5-25.

[6] N. G. Hastopoulos, J. P. Donoghue. The science of neural interface systems. Annu. Rev. Neurosci., 32 (2009), 249-266.

[7] L. R. Hochberg. Turning thought into action. New. Eng. J. Med., 359 (2008), 1175-1177. 
[8] S-P. Kim, J. Simeral, L. Hochberg, J. P. Donoghue, M. J. Black. Neural control of computer cursor velocity be decoding cortical spiking activity by humans with tetraplegia. J. Neurol Engn., 5 (2008), 455-476.

[9] T. A. Kuiken, L. A. Miller, R. D. Lipschutz, B. A. Luck, K. Stubblefield, P. A. Marasco, P. Zhou, G. A. Dumanian. Targeted reinervation for enhanced prosthetic arm function in a woman with a proximal amputation: a case study. Lancet, 369 (2007), 371-380.

[10] J. Ma, J. Wu. Patterns, memory and periodicity in two-neuron delayed recurrent inhibitory loops. Math. Model. Nat. Phenom., 5 (2010), 67-99.

[11] J. Milton, P. Jung. Epilepsy as a dynamic disease. Springer, New York, 2003.

[12] J. Milton, J. Foss. Oscillations and multistability in delayed feedback control. In: Case Studies in Mathematical Modeling: Ecology, physiology and cell biology (H. G. Othmer, F. R. Adler, M. A. Lewis, J. C. Dallon, eds). Prentice Hall, Upper Saddle River, NJ, 1997, pp. 179-198.

[13] J. Milton, P. Naik, C. Chan, S. A. Campbell. Indecision in neural decision making models. Math. Model. Nat. Phenom., 5 (2010), 125-145.

[14] L. Modolo, R. Edwards, J. Campagnaud, B. Bhattacharya, A. Beuter. Past, present and future of brain stimulation. Math. Model. Nat. Phenom., 5 (2010), 185-207.

[15] M. M. Monti, A. Vanhaudenhuyse, M. R. Coleman, M. Boly, J. D. Pickard, L. Tshibanda, A. M. Owen, S. Laureys. Willful modulation of brain activity in disorders of consciousness. New Eng. J. Med., 362 (2010), 579-589.

[16] I. Osorio, M. G. Frei. Real-time detection, quantification, warning, and control of epileptic seizures: The foundations for a scientific epileptology. Epilepsy Behav., 16 (2009), 391-396.

[17] P. M. Pardalos, J. C. Sackellares, P. R. Carney, L. D. Iasemidis, eds. Quantitative Neuroscience: Models, algorithms, diagnostics and therapeutic applications. Kluwer Academic Publications, New York, 2004.

[18] A. A. Sharp, M. B. O’Neil, L. F. Abbott, E. Marder. The dynamic clamp: artificial conductances in biological neurons. Trends Neurosci., 16 (1993), 389-394.

[19] W. Singer. A versatile code for the definition of relations?. Neuron, 24 (1999), 49-65.

[20] L. Stark. Environmental clamping of biological systems: Pupil servomechanism. J. Opt. Soc. Amer., 52 (1962), 925-930.

[21] P. A. Tass. Phase Resetting in Medicine and Biology: Stochastic modelling and data analysis. Springer Series in Synergetics, New York, 1999. 
[22] Y. Yarom. Rhythmogenesis in a hybrid system interconnecting an olivary neuron to an analog network of coupled oscillators. Neurosci., 44 (1991), 263-275. 